\title{
Pro-activity in Collaborative Service Ecosystems
}

\author{
Tiago Cardoso and Luis M. Camarinha-Matos \\ Faculty of Sciences and Technology, \\ Universidade Nova de Lisboa / Uninova, Portugal \\ $\{$ tomfc, cam\}@uninova.pt
}

\begin{abstract}
Service Orientation has been an extensively used approach to model the services Collaborative Networks' $(\mathrm{CN})$ members are willing to provide to customers. The evolution of this paradigm has followed an improvement path since its early forms. Nevertheless bottlenecks still exist, namely in what concerns: 1) a gap between the business and the information and communication technology perspectives; 2) an adequate Quality of Service (QoS) assessment mechanism for the $\mathrm{CN}$ context. As a contribution to these issues, this paper proposes: 1) the creation of a collaborative Service Ecosystem, introducing pro-activeness elements towards an auto-initiative representation of $\mathrm{CN}$ member's services; 2) the introduction of a QoS mechanism needed to facilitate the assessment of services in a $\mathrm{CN}$ context.
\end{abstract}

Keywords: Pro-Active Service Entity, Conspicuity, Service Ecosystem.

\section{Introduction}

Service Orientation is, perhaps, the most commonly used approach for Collaborative Network's (CN) support systems in the existing case studies and proof of concept prototypes. Nevertheless, although this approach had a considerable evolution in the last decade, there still exist bottlenecks that act as inhibitors for a larger scale adoption of Service Oriented Architectures (SOA) in the CN context [1, 2].

On one hand, business and Information and Communication Technology (ICT) perspectives still diverge on the notion of service. Under the business perspective, entities deal with resource management, quality of service or client satisfaction issues. From the ICT perspective, the focus is on the definition of standard communication protocols, namely in what concerns remote method invocation, or information exchange formats among distinct systems, towards solving interoperability issues [3].

As discussed in [4], "the myth of open web-services" within a market without rules, as expected at the beginning, turned out not to be a realistic "dream". The main reason pointed out is that instead of an open marketplace of competing products or services, "it's about branding and simplicity: people want simplicity and quality rather than choices". The authors refer to the appearance of "industrial Service Parks" as the foreseen realistic approach instead of open independent Services spread around the Internet. Such structures "will offer sets of web-services with their own sets of rules for combining and modifying" the services. 
On the other hand, from the industry perspective as presented in [5], for example, next-generation systems, applications and services will raise opportunities and challenges based on their intelligence.

Finally, the evolution of the Services paradigm and industry practices point out the growing importance of customer interaction, as presented in [6], other than just assuring low cost, product performance and high quality, for long-term success.

These three factors are the base inspiration for the proposal made in this paper: the Pro-Active Service Ecosystem Framework (PASEF):

- Appearance of Service Parks - PASEF is a form of a delimited service park;

- Services Intelligence - the introduction of pro-activeness fosters one aspect of the implementation of intelligence at the service level.

- Client / customer interaction - based on pro-activeness, it becomes possible to develop a new QoS assessment mechanism that provides accurate and up-to-date information. Based on this mechanism, a method for the systematic client satisfaction information retrieval within PASEF is also introduced.

\section{Service Orientation Evolution}

Service Orientation, as well as the particular case of Web-Services, have experienced considerable evolution in the last decade. In the early forms of Web-Services, during the late 90s, the keyword was "publish" and the technology and standards provided the means needed to put Web-Services available through the Internet, expecting a worldwide range of new potential clients. As time proved, these worldwide potential new clients' benefit did not happen. Only big companies took advantage of this new approach, based on their marketing machinery. The SME Web-Services did not benefit mainly because they were not known by such worldwide potential client set.

Around 2002, the Web-Services' registries, like the case of UDDI, were added in order to solve this problem. Nevertheless, in the SME context, although the technological capabilities brought by UDDI introduced the possibility of being found, there still was no big change, given the lack on guarantees or trust requested by clients, among other factors, like the lack of specialists for development purposes. Nevertheless, at that time, technologically speaking, many web-services became able to be "plugged in", called and invoked - ready for the next stage that was the composition of distinct Web-Services, provided by disperse entities. The Service Oriented Architectures (SOA) appeared around 2006, introducing tools and mechanisms to automate web-services' composition.

More recently, in 2008, Franco et al. [7] proposed the Service Entity concept, introducing a first notion of an aggregation mechanism for distinct Web-Services provided by the same entity. Franco's proposal groups information concerning a service provider plus the services provided, all within the same construct - the Service Entity.

Fig. 1 represents a summary of the main perspectives of the Web-Service evolution towards the actual situation. Although this evolution brought sound improvements, compared to the early forms of Web-Services, there still exist bottlenecks that act as inhibitors for a wider usage of services, which can also be seen as a challenge for the creation of new solutions. Some of these bottlenecks are: 
1. Passiveness - WebServices are passive entities in the sense they stay still, waiting for a client side initiative. Although some pioneer initiatives connect SOA with some auto-initiative approaches, like the Multi-Agent Systems presented

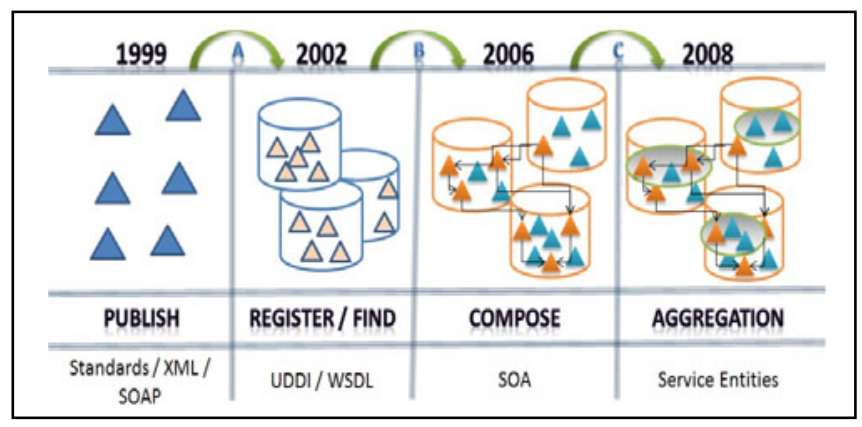

Fig. 1. Web-Service evolution in the examples [8], [9] and [10] still there is no standard integrated approach yet, that could benefit from the strengths of both worlds: MAS \& SOA.

2. Functional restriction - Web-Services do not directly cope with other nonfunctional elements, like the provision of a business process model or a touristic multimedia content, or even some intelligent content provided by an enterprise.

3. Aggregation - although Franco proposed a first approach for the aggregation of services provided by the same entity, all the Web-Service machinery is built on top of previous approaches, inspired in the remote procedure calling era, that is not an integrated approach considering the composition of distinct procedures forming a higher level one. In fact, although service registration in directories may already be made in an integrated manner, registering a set of services at once; the following queries made by clients to such repositories are made on the basis of one query for each service, resulting in independent replies. The fact that an entity may provide two services needed for a given business opportunity (BO) is not considered and thus no advantages may be taken from it.

\section{Pro-Active Service Ecosystem Framework}

The Pro-Active Service Ecosystem Framework (PASEF) proposed in this paper follows the service orientation paradigm evolution through the introduction of two novel aspects: 1) representatives of CN members' services, playing an "ambassador" role through the introduction of pro-activeness; 2) new Quality of Service mechanism, taking benefit from the pro-activeness, towards accurate and up-to-date data, concerning $\mathrm{CN}$ members QoS information.

PASEF comprises 4 groups of concepts (Fig. 2):

1. Service Ecosystem related concepts (SES) - the blocks for the construction of a service taxonomy built for the members of a Service Ecosystem to follow as a guide for interoperability purposes.

2. Membership Modeling related concepts (MM) - the blocks needed to model concrete entities and their services, within a collaborative Service Ecosystem. 
3. Quality of Service related concepts (QoS) - the blocks needed to support QoS assessment, as well as clients' satisfaction that will serve as a base for service selection.

4. Business Process Modeling (BPM) - the blocks that model business processes associated with BOs within PASEF, as well as Proposals and / or suggestions $\mathrm{CN}$ members may submit in order to participate in such BOs.

The Service Ecosystem related concepts are the first ones to be instantiated by the Service Ecosystem initiators, in order to define a common set of rules and

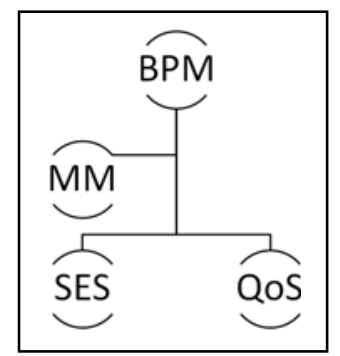

Fig. 2. PASEF Conceptual Groups mechanisms that will guide the $\mathrm{CN}$ members that join this structure. These definitions have to include not only a common terminology, but also standard skeletons for the services that may be provided within that Service Ecosystem. This group includes three concepts with the following definitions:

$>$ D.1 - Meta-Service - the skeleton definition of a service, including details needed to specify service provision proposals or suggestions, both coping with functional services or intelligent-content provision services.

$>$ D. 2 - Service Category - a group of distinct Meta-Services.

$>$ D.3 - Service Taxonomy - the concept that comprises distinct Service Categories and the corresponding Meta-Services.

The Membership Modeling related concepts group is composed of 5 concepts:

D. 4 - Service - the association of a specific CN member and a Meta-Service, including all particular service provision conditions. This concept also includes service connections, gathering the know-how from providers, identifying other Meta-services that usually are requested together with a specific Service.

$>$ D.5 - Service Entity (SE) - the concept that aggregates distinct services provided by the same $\mathrm{CN}$ member, that is useful namely to create proposals and / or suggestions composed of more than one service. In such composed situations promotional conditions may be included for the cases where all the services from that proposal / suggestion become selected.

$>$ D.6 - Pro-Active Service Entity (PSE) - a Service Entity element extended with the ability to behave in a pro-active manner, for example, towards finding new business opportunities or improving the chances that the represented services have to be selected among competitors in a given business opportunity.

$>$ D.7 - Behavior Definition - concept for the configuration of PSE elements including a triggering mechanism; pre-conditions, post-conditions and the specification of a workflow of base actions that the PSE will perform towards the desired goals.

$>$ D.8 - Service Ecosystem - the "space" that brings together all the CN members that are willing to collaborate in an environment created to support and foster the collaborative activity. This Ecosystem performs monitoring functionality, tracking all business opportunities from their early stages through their conclusion phase in order to provide accurate and up-to-date data for clients, concerning CN members' performance, helping them to make their choices whenever they have to select a particular provider for a service they need. 
The Quality of Service mechanism proposed in this paper is based on three concepts:

D.9 - QoS Characteristic - the atomic concept modeling data that may be measured, concerning some property of a service, the provider or the provision itself.

D.10 - QoS Criteria - the combination of a relevant set of QoS characteristics and an evaluation schema for each.

D.11 - Client Satisfaction - the classification of a given service provision under a specific QoS Criteria.

Through the usage of the first two concepts, it is possible to build up specific QoS assessment schemas within a Service Ecosystem. The client satisfaction should then be expressed in an automated manner every time a service provision takes place, in order to feed up PASEF in terms of accurate and up-to-date QoS data. In this way, high QoS from CN members is rewarded with a better selection probability.

Finally, the Business Process Modeling conceptual group is composed of 5 concepts:

$>$ D.12 - Abstract Business Process Model (absBPM) - specification of a graph composed of Meta-Services, transitions and the data included in a process - a workflow model. It is abstract because it does not include service performers yet.

D.13 - Call for Proposals (CfP) - including a list of needed services and provision conditions to which the proposals or suggestions should be posted.

$>$ D.14 - Proposal / Bid - the reply to a CfP including the set of services that a CN member (proposer) intends to provide, as well as a set of provision conditions.

D.15 - Additional Services Suggestion - based on the service connections included in the Service concept, from the Membership Modeling related concepts; providers may suggest the inclusion of additional services. If, for example, a Senior Professional (SP) provides a consultancy service (1) "evaluation of a BPM", it may be the case that SP also provides two other services: (2) "find similar case study BPMs", (3) "compare BPMs". In this situation, SP may connect these services if he or she thinks they make sense being together and, as a result, a suggestion is made by the PSE, for the inclusion of (2) and (3) whenever a call for proposals includes (1).

D.16 - Executable Business Process Model (eBPM) - after an absBPM is created, $\mathrm{CfP} \mathrm{a}$ is made. Based on the received proposals and / or suggestions the absBPM evolves and becomes an eBPM through the eventual inclusion of some suggestions in the workflow and the selection of the proposals that best fit the client's needs. An eBPM may be in one of two states: 1) complete, meaning that all services have a not-empty set of accepted service provision proposals, or 2) ready, meaning that all services that receive transitions from the start point of the workflow model have a not-empty set of accepted provision proposals, i.e., the start activities have at least one possible performer in order for the model execution to start. In this special case, the eBPM will eventually become complete afterwards, during its execution.

Table 1 shows the formal definitions of these 16 concepts: 
Table 1. Summary of PASEF formal definitions

\begin{tabular}{|c|c|c|c|}
\hline $\begin{array}{l}\quad<\mathrm{N}, \mathrm{R}, \mathrm{D}, \mathrm{SC}> \\
\mathrm{N} \text { - Service Name } \\
\mathrm{R} \text { - Reference to } \\
\text { Implementation } \\
\text { D - Description / } \\
\text { service info. } \\
\text { SC - Serv. Category }\end{array}$ & $\begin{array}{l}\text { 2.Service Category } \\
\text { Meta-Services group } \\
\quad<\mathrm{N}, \mathrm{D}, \mathrm{T}> \\
\mathrm{N} \text { - Category Name } \\
\text { D - Category } \\
\text { Description } \\
\mathrm{T} \text { - Services } \\
\text { Taxonomy }\end{array}$ & $\begin{array}{l}\text { 3.Service Taxonomy } \\
\text { Group of Service } \\
\text { Categories. } \\
\qquad \quad<N, D, V> \\
\text { N - Taxon. Name } \\
\text { D - Taxonomy } \\
\text { Description } \\
\text { V - Version info. }\end{array}$ & $\begin{array}{l}\quad \text { 4.Service } \\
\text { Single service } \\
\text { provided by a CN } \\
\text { member } \\
\text { < M, MS, SPC, SC > } \\
\text { M - CN Member id } \\
\text { MS - Meta-Service } \\
\text { SPC -Prov. Conds. } \\
\text { SC - Connection Set }\end{array}$ \\
\hline \begin{tabular}{l}
\multicolumn{1}{c}{ 5.Service Entity } \\
All services plus \\
attributes from a CN \\
member \\
$\quad$ < M, ATS, SS > \\
M - CN Member id \\
ATS - CN member \\
Attribute set \\
SS - provided Service \\
Set
\end{tabular} & 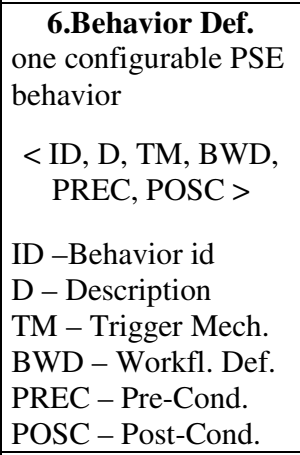 & \begin{tabular}{l}
\multicolumn{1}{c}{ 7.PSE } \\
CN member's service \\
ambassador or \\
representative \\
$\quad<$ SE, BD > \\
SE - Service Entity \\
BD - Behavior \\
Definition set
\end{tabular} & $\begin{array}{l}\text { 8.Service Ecosystem } \\
\text { CN members' space } \\
\text { < ST, PS, BO, PM, } \\
\quad \text { CR, BF > } \\
\text { ST - Service } \\
\text { Taxonomy } \\
\text { PS - PSE set } \\
\text { BO - BO set } \\
\text { PM -Perform. info } \\
\text { CR - Certif. Info }\end{array}$ \\
\hline $\begin{array}{l}\text { 9.QoS Charact. } \\
\text { Measurable property, } \\
\text { from service, provider } \\
\text { or provision } \\
<\mathrm{N}, \mathrm{IM}, \mathrm{MC}> \\
\mathrm{N}-\mathrm{QoS} \text { id } \\
\mathrm{IM} \text { - Info to measure } \\
\text { MC - Measurement } \\
\text { Category }\end{array}$ & \begin{tabular}{|l}
\multicolumn{1}{|c}{ 10.QoS Criteria } \\
Collection of QoS \\
Charact. and \\
evaluation schema \\
< CS, ES, RC, RF > \\
CS - QoS Ch. Set \\
ES - Eval. Schema \\
RC - Restrict Conds. \\
RF - Overall Rating \\
Formula.
\end{tabular} & $\begin{array}{l}\quad \text { 11.Clientnt } \\
\text { Satisfaction } \\
\text { classification of } \\
\text { service provision } \\
\text { under QoS Criteria } \\
\text { < QoS_Crit, }\{E V\}> \\
\text { QoS_Crit. - Quality } \\
\text { of Service Criteria } \\
\text { EV - Evaluation of } \\
\text { each QoS Ch. }\end{array}$ & \begin{tabular}{l}
\multicolumn{1}{c}{ 12.absBPM } \\
Abstract Business \\
Process Model \\
$\quad<\quad\{(\mathrm{A}, \mathrm{MS})\}$, \\
$\quad$ RD, TS $>$ \\
\\
$\{(\mathrm{A}, \mathrm{MS})\}$ Activity \\
and Meta-Service set \\
RD - Relevant Data \\
Set \\
TS - Transition set
\end{tabular} \\
\hline $\begin{array}{l}\text { BPM - Business } \\
\text { Process Model } \\
\text { S - Serv. requested, } \\
\text { SC - Serv. Category } \\
\text { SPC - Service } \\
\text { Provision Conditions } \\
\text { GPC - General } \\
\text { Provision Conditions }\end{array}$ & $\begin{array}{l}\text { 14.Proposal / Bid } \\
\text { PSE provision } \\
\text { intention proposal } \\
\\
<\{\mathrm{S}, \mathrm{SC}, \mathrm{PC}\}, \mathrm{GPC}> \\
\text { S - Proposed Service, } \\
\text { SC - Serv. Category } \\
\text { PC - Serv. Provision } \\
\text { Conditions, } \\
\text { GPC - General } \\
\text { Proposal Conditions }\end{array}$ & \begin{tabular}{l}
\multicolumn{1}{c}{ 15.Suggestion } \\
PSE provision \\
intention suggestion \\
$\quad<\{$ SS, SSC, BS, \\
BSC, PC $\}$, GSC > \\
\\
SS - Suggested Srv \\
SSC - Sug.Srv.Cat. \\
BS - Base Service \\
BSC - Base Srv.Cat \\
PC - Provision \\
Conditions \\
GSC - General \\
Suggest. Conditions
\end{tabular} & 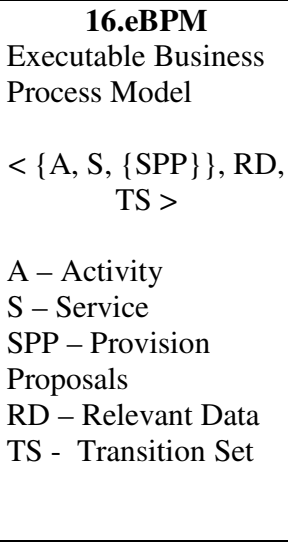 \\
\hline
\end{tabular}


In order to guarantee coherency and consistency in BPMs, a set of rules was defined for the creation of an absBPM (rules i to v) and the following eBPM (rule vi). These rules associate relevant data variables with all the information the services need and produce. The rules also guarantee that all activities are "reachable" and the workflow proceeds after their execution (Table 2).

Table 2. absBPM / eBPM specification rules

\begin{tabular}{|c|c|c|}
\hline$\#$ & Rule & Description \\
\hline $\mathrm{i}$ & $\begin{array}{c}\forall A=\Pi_{1}(A S) \\
A S \in \Pi_{1}(a b s B P M) \\
\exists^{1} \mathrm{MS} \in \mathrm{T}\end{array}$ & $\begin{array}{l}\text { for every activity A of the Activity and Service } \\
\text { Set AS of an absBPM, there exists one and only } \\
\text { one Meta-Service MS from the Service } \\
\text { Ecosystem's Taxonomy T. }\end{array}$ \\
\hline ii & $\begin{array}{c}\forall A=\Pi_{1}(A S) \\
A S \in \Pi_{1}(a b s B P M) \\
\exists \operatorname{Tr}(\mathrm{E}, \mathrm{A}) \in \Pi_{3} \text { absBPM }\end{array}$ & $\begin{array}{l}\text { for every activity A of the Activity and Service } \\
\text { Set AS of an absBPM there exists, at least, one } \\
\text { transition Tr of absBPM from an element E to A } \\
\text { - reach guarantee. }\end{array}$ \\
\hline iii & $\begin{array}{c}\forall A=\Pi_{1}(A S) \\
A S \in \Pi_{1}(a b s B P M) \\
\exists \operatorname{Tr}(\mathrm{A}, \mathrm{E}) \in \Pi_{3}(\mathrm{absBPM})\end{array}$ & $\begin{array}{l}\text { for every activity A of the Activity and Service } \\
\text { Set AS of an absBPM, there exists, at least, one } \\
\text { transition Tr from A to some element E - } \\
\text { proceed guarantee. }\end{array}$ \\
\hline iv & $\begin{array}{c}\forall M S(P)=\Pi_{2}(A S), A S \in \Pi_{1}(a b s B P M) \\
\exists \mathrm{RD} \in \Pi_{2}(a b s B P M) \mid\left\{p_{i}, \mathrm{i} \in \mathbb{N}\right\} \\
\left\{r d_{j}, j \in \mathbb{N}\right\} \forall p_{i} \in \mathrm{P} \exists \mathrm{rd}_{j} \in \mathrm{RD}\end{array}$ & $\begin{array}{l}\text { for each Meta-Service MS with a parameter set } \\
P \text {, there exists a relevant data variable set } R D \text {, } \\
\text { associating a specific variable } \mathrm{rd}_{\mathrm{j}} \text { to each } \\
\text { particular parameter } \mathrm{p}_{\mathrm{i}} \text {. }\end{array}$ \\
\hline $\mathrm{v}$ & $\begin{array}{c}\forall M S(O R)=\Pi_{2}(A S), A S \in \Pi_{1}(a b s B P M) \\
\exists \mathrm{RD} \in \Pi_{2}(a b s B P M) \mid \\
\exists\left\{\operatorname{or}_{i}, \mathrm{i} \in \mathbb{N}\right\},\left\{r d_{j}, j \in \mathbb{N}\right\} \mid \\
\forall o r_{i} \in \mathrm{OR} \exists \mathrm{rd}_{j} \in \mathrm{RD}\end{array}$ & $\begin{array}{l}\text { for each Meta-Service MS with an output result } \\
\text { set OR, there exists a relevant data set } \mathrm{RD} \text {, } \\
\text { associating a specific variable } \mathrm{rd}_{\mathrm{i}} \text {, to each } \\
\text { particular result or }{ }_{i}\end{array}$ \\
\hline vi & $\begin{array}{c}\forall(A, S) \in \Pi_{1}(\text { absBPM }) \\
\operatorname{Tr}(\text { Start, A }) \in \Pi_{3}(\text { absBPM }) \\
\text { SPP } \neq \emptyset\end{array}$ & $\begin{array}{l}\text { for every Activity A associated with a service S, } \\
\text { if there is a transition Tr from the Start point in } \\
\text { the workflow to A, there is a not-empty set of } \\
\text { provision proposals SPP identifying potential } \\
\text { performer of S. }\end{array}$ \\
\hline
\end{tabular}

\section{Application Area - Active Ageing}

PASEF is being applied to a Professional Virtual Community (PVC) of Senior Professionals (SPs). The first article found foreseeing this challenge or need was [11] in 2004, based on the increase of life expectation, as well as the need for sustainable economies. The purpose of this application is to "support active ageing and facilitating better use of the talents and potential of retired or retiring senior professionals", as mentioned in [12]. In fact, three main perspectives can be identified concerning the current early retirement of people in many countries:

1. The retirement age is far from the age when elderly people's working capabilities start decreasing.

2. Many senior professionals prefer to continue working, although under a more flexible schema, instead of starting a process of a lonely experience.

3. The knowledge attained during a life-long experience is an asset that the economy thanks and elderly persons feel glad to share. 
These facts pull the research community to find mechanisms and solutions in order to provide the base for these persons to be able to continue their active life after retirement. There are already many associations of SPs formed towards helping to find solutions. Actually, PASEF development was inspired on the needs identified in contact with two Portuguese associations of senior professionals.

As a result, an implementation of a prototype framework took place, following PASEF specification, composed of 6 modules:

1. PASEF Toolbox - The main objective of the PASEF Toolbox is to enable a fast scenario definition, as well as launch, test, and monitor all the modules form the Pro-Active Service Ecosystem Framework prototype. (Fig. 3)

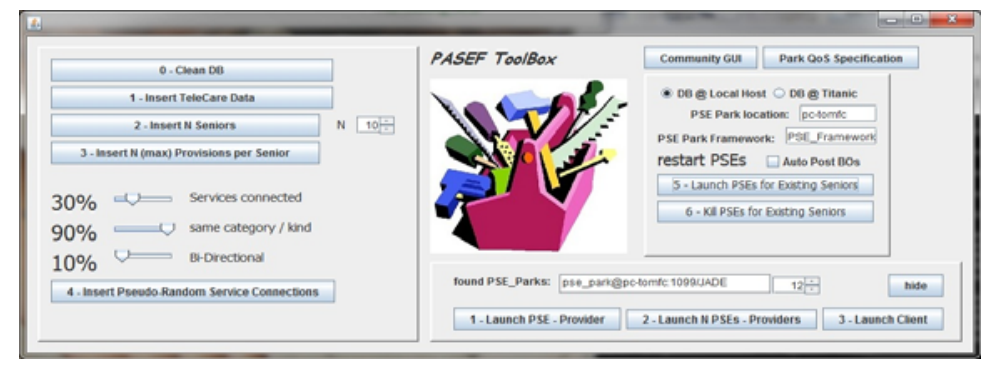

Fig. 3. PASEF Toolbox

2. Service Taxonomy Management - as identified in the Service Ecosystem related concepts group, a module was developed for the specification of a service Taxonomy to which the SPs should comply (Fig. 4 - lower side).

3. Seniors' Community Management - in this case, PASEF is intended to a PVC and a community management module was needed, in order to introduce the SPs that are willing to provide consultancy services where their life-time expertise can be used to keep them active. This module provides the functionality of service specification, as well as a service connections knowledge manifestation, towards the PSE launch, in order to represent the corresponding SP, in an "ambassador" like manner.

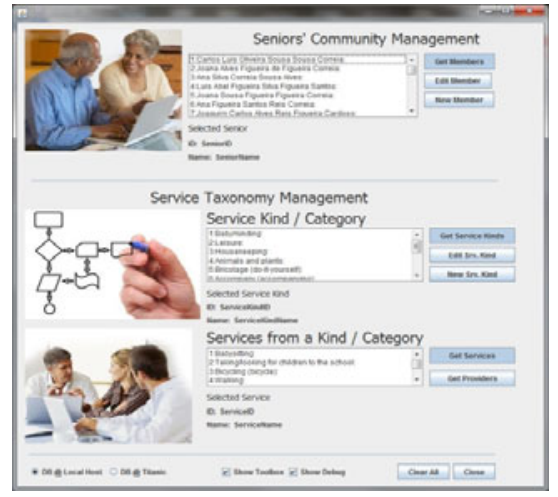

Fig. 4. Service Taxonomy Management \& Service Community Management

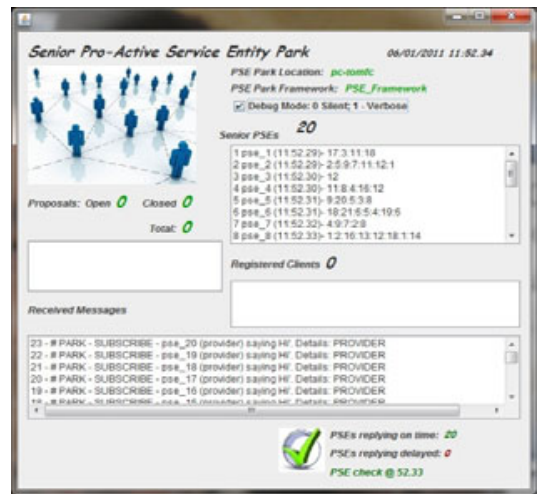

Fig. 5. Senior Pro-Active Service Entity Park 
4. Senior Pro-Active Service Entity Park - the module that monitors the activity of the Ecosystem, showing active PSEs, open Business Opportunities and the messages exchanged among distinct actors of PASEF (Fig. 5).

5. Workflow Editor - The module for the specification of absBPMs and later for the selection of SP performers in order to achieve an eBPM. Fig. 6 represents an example absBPM, without performers, as explained above.

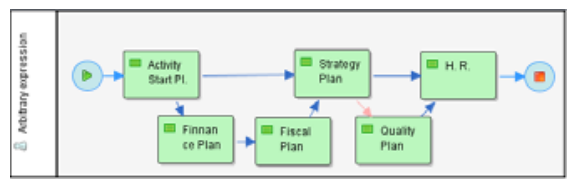

Fig. 6. Example absBPM

6. Workflow Engine - Finally, a workflow engine was needed, in order to launch the execution of the eBPMs. Fig. 7 represents an eBPM that is being executed.

The model of Fig. 7 corresponds to the absBPM of Fig. 6, to which 3 SPs were selected as performers of the consultancy services. The selection of the SPs for each service is supported by the QoS data stored at PASEF. It is interesting to notice that the lines in the model of Fig. 7 correspond to the PSEs representing the selected seniors, following the BPMN standard notation.

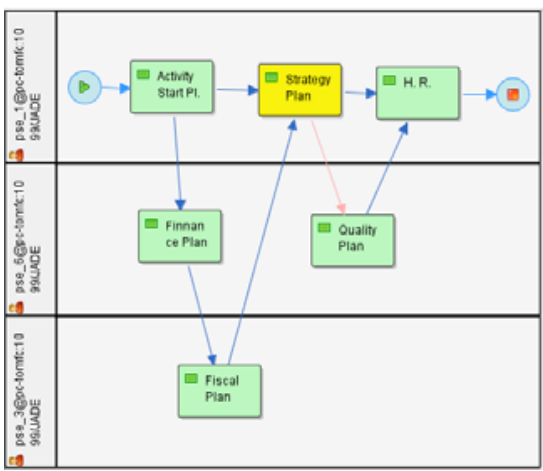

Fig. 7. Example eBPM being executed In this particular example, two PSEs have made successful proposals composed of more than one service. These proposals are made by the PSEs themselves, following a configuration that the corresponding SP gave them, in terms of autonomy.

Besides supporting the professional life of seniors, collaborative networks can also play a relevant role in other life settings, namely independent living, healthy living, and recreation in life, as identified in the BRAID roadmap on ICT and Ageing. In all these areas there is a trend to evolve towards more integrated services, involving multiple stakeholders through well coordinated collaborative ecosystems. PASEF can be applied in such contexts.

\section{Conclusions and Future Work}

The main objective of this work was to define a set of base concepts that better match CN members' expectations concerning the mechanisms they have to model the services they are willing to provide to the network, following the service orientation paradigm evolution. This goal was achieved through the introduction of proactiveness elements and a new QoS Mechanism that benefits from such elements.

The presented concepts allow $\mathrm{CN}$ members to model their services through PSEs, within a well-delimited Ecosystem, and configure them to better represent and 
promote the services they are willing to provide, under an auto-initiative basis, instead of the passiveness of current approaches. Furthermore, the QoS mechanism improves service selection processes towards rewarding the best providers.

Particularly for the case of senior professionals, the prototype developed intends to serve a community or association of elderly people in order to provide them the means to continue their active life after retirement. These people may then configure their PSEs in order to make a good balance of their working periods and the leisure moments, as they will. The PSEs will make all the "ambassador" work for them.

PASEF is the result of a research work that integrates distinct perspectives [1], [3], [13]. This paper presents the formal integrated framework definition of PASEF that served for the development of a proof of concept prototype system. The ongoing work is the validation stage, made through presentations of the prototype to stakeholders from ICT world and Active Ageing associations.

Acknowledgments. This work was supported in part by FCT (CTS multiannual funding) through the PIDDAC Program funds, and BRAID project funded by the European Commission.

\section{References}

1. Cardoso, T., Camarinha-Matos, L.: Pro-Active Asset Entities in Collaborative Networks. In: Camarinha-Matos, L., Pereira, P., Ribeiro, L. (eds.) Emerging Trends in Technological Innovation, pp. 93-102. Springer, Boston (2010)

2. Rabelo, R., et al.: The Ecolead ICT Infrastructure For Collaborative Networked Organizations. In: Network-Centric Collaboration and Supporting Frameworks, pp. 451460. Springer, Boston (2006)

3. Cardoso, T., Camarinha-Matos, L.: Pro-Active Service Entity Framework for a Better Mapping between Business and Software. In: Camarinha-Matos, L., Boucher, X., Afsarmanesh, H. (eds.) Collaborative Networks for a Sustainable World, pp. 451-460. Springer, Boston (2010)

4. Charles, P., Christoph, B.: The Myth of Open Web Services: The Rise of the Service Parks. IEEE Internet Computing 12(3), 94-95 (2008)

5. Jammes, F., Smit, H.: Service-oriented paradigms in industrial automation. IEEE Transactions on Industrial Informatics 1(1), 62-70 (2005)

6. Burianek, F., Bonnemeier, S., Ihlenburg, D.: Solution Selling: The Need for Customer Interaction. In: Alt, R., Francyzk, B., Frahnrich, K.-P. (eds.) Proceedings of International Symposium of Services Science, pp. 127-137 (2009)

7. Franco, R., Ortiz Bas, A., Lario Esteban, F.: Modeling extended manufacturing processes with service-oriented entities. Service Business 3(1), 31-50 (2009)

8. Svirskas, A., et al.: Virtual Organization Management Using Web Service Choreography And Software Agents. In: Network-Centric Collaboration and Supporting Frameworks, pp. 535-542. Springer, Boston (2006)

9. Petersen, S.A., Rao, J., Matskin, M.: Virtual Enterprise Formation Supported by Agents and Web Services. In: Protogeros, N. (ed.) Agents and Web Service Technologies in Virtual Enterprises (2008) 
10. Brazier, F.M.T., et al.: Agents and Service-Oriented Computing for Autonomic Computing: A Research Agenda. IEEE Internet Computing 13(3), 82-87 (2009)

11. Camarinha-Matos, L., et al.: Emerging Collaborative Forms. In: Collaborative Networked Organizations, pp. 41-64. Springer, Heidelberg (2004)

12. Camarinha-Matos, L., Afsarmanesh, H.: Active Ageing Roadmap - A Collaborative Networks Contribution to Demographic Sustainability. In: Camarinha-Matos, L., Boucher, X., Afsarmanesh, H. (eds.) Collaborative Networks for a Sustainable World, pp. 46-59. Springer, Boston (2010)

13. Cardoso, T., Camarinha-Matos, L.M.: Pro-Active Service Entities - Improving Selection Chances within large Senior Professional Virtual Community scenario (2011) 